\title{
Heart Failure Clinics Are Still Useful (More Than Ever?)
}

Tiny Jaarsma and Anna Strömberg

\section{Linköping University Post Print}

\section{Tweet}

N.B.: When citing this work, cite the original article.

Original Publication:

Tiny Jaarsma and Anna Strömberg, Heart Failure Clinics Are Still Useful (More Than Ever?), 2014, Canadian Journal of Cardiology, (30), 3, 272-275.

http://dx.doi.org/10.1016/j.cjca.2013.09.022

Copyright: Elsevier

http://www.elsevier.com/

Postprint available at: Linköping University Electronic Press

http://urn.kb.se/resolve?urn=urn:nbn:se:liu:diva-105745 
Point/Counterpoint: Heart Failure Clinics Are No Longer Useful

Title: Heart Failure Clinics Are Still Useful (more than ever?)

Tiny Jaarsma and Anna Strömberg

Authors:

Tiny Jaarsma, RN PhD, Professor, Department of Social and Welfare studies, Faculty of Health Sciences, Linköping University, Linköping, Sweden. *

Anna Strömberg, RN, PhD Professor, Department of Medicine and Health Sciences, Division of nursing, Faculty of Health Sciences Linköping University, Linköping, Sweden and Department of Cardiology UHL, County Council of Östergötland, Linköping, Sweden.

* Corresponding author: tiny.jaarsma@liu.se 


\begin{abstract}
Heart Failure (HF) clinics have had an important role in optimal HF management and the effectiveness of these clinics has been studied intensively. A HF clinic is one of the various ways to organize a HF disease management program. There is good evidence that HF disease management can improve outcomes in HF patients, but it is not clear what the optimal components of these programs are and what the relative effectiveness of a HF clinic is compared to other forms of HF management. After initial positive reports on the effect of $\mathrm{HF}$ clinics, these clinics were implemented in a lot of countries, although in different formats and of varying quality. In this paper we describe the initial need for HF clinics, reflect on their development over time and discuss the role of HF clinics in the current need for HF disease management.
\end{abstract}




\section{Introduction:}

Heart failure (HF) is a major challenge in current health care with an increasing number of patients worldwide and with the aging of the population, the impact of $\mathrm{HF}$ is expected to increase $(1,2)$. HF is an important contributor to health care costs, with more elderly people hospitalized due to HF than any other medical diagnosis. Based on a large body of scientific evidence, current HF guidelines stress the importance of a multifaceted approach to HF management consisting of optimal diagnosis, pharmacological, device and nonpharmacological treatment, including life style advice, optimal transition and coordination of care $(1,2)$. Along the HF disease trajectory the condition of the patient often changes, symptoms may progress over time and in most patients the course is unpredictable, with phases of crisis, chronicity and the terminal stages. HF patients often have several exacerbations during their disease trajectory, which are mostly accompanied by rehospitalizations or extra visits to a health care professional, resulting in high costs in health care and a negative impact on the quality of life of patients and their families $(3,4)$. A HF clinic can play an important role in optimal HF management and the concept of these clinics has been studied intensively in the past years $(5,6)$. A HF clinic can be defined as a unit providing access to multidisciplinary teams including specialist HF nurses and physicians delivering advanced diagnostic or treatment services (6), in hospital or in primary care. HF clinics are a tool for delivering care according to clinical guidelines and providing advanced diagnostic or treatment services. (6) With newer forms of HF management in home care or primary care or by telemonitoring, one wonders if there is still a place for HF clinics and if they suit the state of the health care, patients' wishes and costs.

This is especially relevant, since a recent Cochrane analysis (7) concluded that although there is now good evidence that case management type interventions led by a HF specialist nurse improve outcomes in HF patients, they found it not possible to say that the HF clinic is superior to other forms of HF management. One might also argue that with the improving trends in the long-term prognosis after acute HF $(3,5)$ with declining overall 1-year mortality rate, these specialized HF clinics are not needed anymore. However, before throwing out the baby with the bathwater, we would like to describe the initial need for HF clinics, reflect on their development over time and discuss the role of HF clinics in the current need for HF disease management.

\section{Phase 1: First need and development}


The first HF disease management programs were established in the 1990's as a solution to the high readmission rate of patients and their poor prognosis. Readmissions were often seen as preventable and were related to non-adherence, inadequate medical treatment or inadequate reaction of patients and health care providers to deterioration. HF clinics in the first landmark studies included several components such as patient education, optimization of medication, and close follow-up, either in outpatient HF clinics or at home (8-11). In the years after these first positive reports, HF management programs were increasingly evaluated and implemented in several countries worldwide. Meta-analysis confirmed the effectiveness of HF disease management programs that were often hospital based, with care delivered at outpatient clinics, sometimes with outreach to patients' homes by HF nurses.(12)

\section{Phase 2: Implementation and reflection}

After the first successful trials and positive meta-analysis, major guidelines recommended HF management programs for recently hospitalized HF patients and for other high-risk patients. $(1,2)$. As delivery of care varies in different health care systems worldwide, the organization of a HF management program was advised to be based on patient needs, financial resources, available personnel, and administrative policies and adapted to local priorities and infrastructure (13), implying that it is difficult to prescribe one optimal format across the whole world. Different models might have advantages and disadvantages that might be more applicable to some countries then to others. (Table 1). In the course of time, an increasing number of meta-analysis and first cost effectiveness studies led to further implementation of HF programs across the world. However, substantial disparity in access to HF care was found internationally, nationally and also regionally, with a large range in the complexity of services offered. (14,15) A wide range of models was offered. However, the most common model was hospital-based and specialty-only resulting in a limited availability to the oldest, frailest and those with multi-morbidity, in almost every country. For example, in a European survey we found that half of the programs were located in outpatient clinics and others as home-based programs (15). Furthermore, even if HF clinics are available, the referral to and subsequent enrollment in HF clinics is far from optimal $(14,16)$ with estimation that one-seventh of HF patients were referred to an HF clinic (14), suggesting that implementation is far from optimal.

Meanwhile, although most HF management programs aim at optimization of both pharmacological and non-pharmacological management, the most optimal model for HF 
management is not known. Recent large-scale studies show that not all models are equally successful to improve outcomes $(17,18)$. These results indicate that a sophisticated approach to HF management is needed and questions remain on the optimal 'dose' and format of follow-up.

\section{Phase 3: Current state and future challenges}

Several challenges remain in optimal care delivery and the HF management programs need to consider issues related to the place of delivery, quality and new patient groups. In addition, HF clinics need to be adaptive for patients over time as they will need more or less of the components in a HF clinic during their HF trajectory

- Place of delivery: clinic or/and home?

The recent Cochrane analysis (7) reviewed clinical service organization for HF patients by analyzing 25 trials (5,942 people) and it was concluded that it is not possible to say what the optimal components of these HF management interventions are, either in the form of a HF clinic or other formats. As also described in the recent WHICH? trial (19), none of these interventions is clearly superior. At the same time, it was found that stable HF patients on optimal medical therapy who received regular follow-up in a well-organized primary care system in Denmark had similar outcomes compared to those who received long-term followup in a specialized HF clinic. (20) This indicates that in some health care systems home visits might imply lower health care costs, while in another study in the Netherlands, a HF clinic seemed more cost effective than cardiology care only or a very intensive program including home visits (21). We believe that the location for follow-up is not the main concern; rather it is the quality, structure, components and availability of the follow-up that is important.

- Quality improvement

Currently, quality of care varies considerably between hospitals and across countries and regions. Quality improvement programs have proved successful in enhancing the level of care for HF patients (22). Considering the huge variation in patients enrolled and services provided between different HF clinics there is a significant need to develop guidelines and guidance documents for the organisation and deliverables of HF clinics. (23) Furthermore, even in the era of HF clinics there is wide variability in HF self-care across countries and there is markedly poor adherence to most self-care behaviors regardless of country. In a study where data on self-care were pooled from 5964 patients with HF from Europe, Australia, Asia, and 
North and South America, self-care behaviors were found to be sub-optimal. Most patients reported a high adherence to their pharmacological treatment, but adherence to exercise and weight monitoring was low. The level of self-care regarding taking an annual flu shot and following a low sodium diet varied most across the countries. HF clinics have been found to improve self-care behaviors (11).

- New patient groups

HF clinics were initially established to reduce morbidity and mortality, and they focused their attention on patients at the more severe end of the spectrum. In contrast, there are few data addressing the benefit of HF services in the earlier stages of HF. It might be advocated that all patients with HF should be able to access the benefits of organized HF care. (24). It is also advocated to have an organization of care that includes the whole HF illness trajectory and phases of crisis, chronicity and the terminal stages. The crisis phase refers to the period between the prediagnostic phases when symptoms start occurring to the initial period after the diagnosis when the patient starts adapting to the disease. The chronic phase is defined as the period between the initiation of treatment and the transition to the terminal stage of the illness. Each phase is associated with different demands (25). However, there are several patient groups that remain challenging to include in HF clinics. Unfortunately, patients with the greatest needs often lack optimal treatment and care. For example, patients in need of palliative care are not always included in HF clinics in an optimal way. A comprehensive approach is needed including access to home care and collaboration with specialized palliative care units. Furthermore, complex patients with multi-morbidities and co-morbidities are not always optimally treated in a specialist HF clinic since there are often many caregivers involved with challenges in coordination and communication.

In case of comorbidity and/or multi-morbidity it may be desirable to involve other medical specialties in the disease management program of patients with HF. It differs from patient to patient with some without comorbidity and others with extensive multi-morbidity with varying consequences for the HF treatment and care. Depending on the on the stability of the patient, the success of optimization of HF medication and self-care and on available resources to optimally manage a HF patient with other diseases outside the HF clinic, some patients might be discharged from the HF clinic after initial optimization and be followed by other specialists. Other patients might have a continuing follow-up in the HF clinic and the staff will take a more case management approach with the care. Patients with multiple chronic 
conditions can benefit from an individualized treatment plan based on components from different disease management programs involving not only at HF care but also care for all other comorbidities. (26)

\section{Final reflection: Heart Failure Clinics Are STILL Useful (more than ever?)}

In general, management of HF patients is far from optimal at this moment. Although clear guidelines on diagnosis and treatment are available, patients are not always diagnosed in time and not all patients receive optimal medical treatment or non-pharmacological advice. Major gains can still be made, especially with regard to symptom monitoring and increasing exercise. In addition, structured follow-up is needed along the disease trajectory and early intervention in case of deterioration is vital to prevent readmission or death. When available and accessible, the multidisciplinary model of care is the preferred choice and this may take place in a specialized HF clinic. The current challenge, however, is to find an optimal model of organization that is cost effective in taking care of the growing group of HF patients and addressing their specific needs. Therefore, creative models are needed, such as 'light' and 'plus' versions, home based or hybrid primary care based /hospital based programs. In this range of models, the 'classic' HF clinic still has a place, either in its old form or in extending its services with new components such as telemonitoring or tele-education. 



\section{References}

1. McMurray JJ, Adamopoulos S, Anker SD, et al. ESC Guidelines for the diagnosis and treatment of acute and chronic heart failure 2012: The Task Force for the Diagnosis and Treatment of Acute and Chronic Heart Failure 2012 of the European Society of Cardiology. Eur J Heart Fail 2012 ;14:803-69.

2. Arnold JMO, Howlett JG, Ducharme A, et al. Canadian Cardiovascular Society Consensus Conference guidelines on heart failure - 2008 update: Best practices for the transition of care of heart failure patients, and the recognition, investigation and treatment of cardiomyopathies. Can J Cardiol. 2008; 24: 21-40.

3. Park D, McManus D, Darling C, et al. Recent trends in the characteristics and prognosis of patients hospitalized with acute heart failure. Clin Epidemiol. 2011; 3: 295-303.

4. Jaarsma T, Beattie JM, Ryder M, et al. Palliative care in heart failure: a position statement from the palliative care workshop of the Heart Failure Association of the ESC. Eur J Heart Fail 2009;11:433-43.

5. Savard LA, Thompson DR, Clark AM. A meta-review of evidence on heart failure disease management programs: the challenges of describing and synthesizing evidence on complex interventions. Trials. 2011; 12: 194.

6. Thomas R, Huntley A, Mann M, et al. Specialist clinics for reducing emergency admissions in patients with heart failure: a systematic review and meta-analysis of randomised controlled trials. Heart. 2013 Feb;99(4):233-9.

7. Takeda A, Taylor SJ, Taylor RS,et al. Clinical service organisation for heart failure. Cochrane Database Syst Rev. 2012 Sep 12;9.

8. Rich MW, Beckham V, Wittenberg C, et al. A multidisciplinary intervention to prevent the readmission of elderly patients with congestive heart failure. $\mathrm{N}$ Engl $\mathbf{J}$ Med 1995;333:1190-5.

9. Stewart S, Marley JE, Horowitz JD. Effects of a multidisciplinary, home-based intervention on unplanned readmissions and survival among patients with chronic congestive heart failure: a randomized controlled study. Lancet 1999;354:107783.

10. Jaarsma T, Halfens R, Huijer Abu-Saad H, et al. Effects of education and support on self-care and resource utilization in patients with heart failure. Eur Heart J 1999;20:673-82. 
11. Stromberg A, Martensson J, Fridlund B, et al. Nurse-led heart failure clinics improve survival and self-care behaviour in patients with heart failure. Eur Heart $\mathbf{J}$ 2003;24:1014-23.

12. McAlister FA, Stewart S, Ferrua S, McMurray JJ. Multidisciplinary strategies for the management of heart failure patients at high risk for admission: a systematic review of randomized trials.J Am Coll Cardiol 2004;44:810-9.

13. Dickstein K, Cohen-Solal A, Filippatos G, et al. ESC guidelines for the diagnosis and treatment of acute and chronic heart failure 2008. Eur J Heart Fail 2008; 10:93389.

14. Gravely S, Ginsburg L, Stewart DE, Mak S, Grace SL Referral and use of heart failure clinics: what factors are related to use? Can J Cardiol 2012;28:483-9.

15. Jaarsma T, Stromberg A, De Geest $S$, et al. Heart failure management programmes in Europe. Eur J Cardiovasc Nurs 2006;4:197-205.

16. Howlett JG, McKelvie RS, Costigan J et al. The 2010 CCS guidelines for the diagnosis and management of heart failure update: Heart failure in ethnic minority populations, heart failure and pregnancy, disease management, and quality improvement/assurance programs. Can J Cardiol 2010;26:185-202.

17. Jaarsma T, Wal MHL van der, Lesman-Leegte I, et al. Effects of moderate and intensive disease management program on outcome in patients with heart failure. The Coordinating Study evaluating Outcomes of Advising and Counselling in Heart Failure (COACH). Arch Intern Med 2008;168:316-24.

18. Angermann CE, Störk S, Gelbrich G, et al. Mode of action and effects of standardized collaborative disease management on mortality and morbidity in patients with systolic heart failure: the Interdisciplinary Network for Heart Failure (INH) study. Circ Heart Fail. 2012 Jan;5(1):25-35.

19. Stewart S, Carrington MJ, Marwick TH, et al.Impact of home versus clinic-based management of chronic heart failure: the WHICH? multicenter, randomized trial. J Am Coll Cardiol. 2012;60(14):1239-48.

20. Schou M, Gustafsson F, Videbaek L, et al .Extended heart failure clinic follow-up in low-risk patients: a randomized clinical trial (NorthStar). Eur Heart J. 2013 Feb;34(6):432-42.

21. Postmus D, Pari AA, Jaarsma T, Luttik ML, van Veldhuisen DJ, Hillege HL, Buskens E. A trial-based economic evaluation of 2 nurse-led disease management programs in heart failure. Am Heart J 2011;162:1096-104. 
22. Fonarow GC, Abraham WT, Albert NM, et al. Factors identified as precipitating hospital admissions for heart failure and clinical outcomes: findings from OPTIMIZE-HF. Arch Intern Med 2008;168(8):847-54.

23. Abrahamyan L et al, Insights into the contemporary management of heart failure in specialized multidisciplinary ambulatory clinics. Can J Cardiol. 2013 Sep;29(9):1062-8.

24. McDonagh TA, Blue L, Clark AL, et al.ESC Heart Failure Association Standards for delivering heart failure care. Eur J Heart Fail 2011;13:235-41

25. Rolland, JS. Chronic illness and the life cycle; A conceptual framework. Family Process 1987:26:203-21.

26. Annema C, Luttik ML, Jaarsma T. Do patients with heart failure need a case manager? J Cardiovasc Nurs 2009;24:127-31 